May 1998

IFP-758-UNC

\title{
Bileptons from Muon Collider Backward Scattering.
}

\author{
Paul H. Frampton and Xiaohu Guan \\ Department of Physics and Astronomy, \\ University of North Carolina, Chapel Hill, NC 27599-3255
}

\begin{abstract}
There are serious discussions for building a muon collider with $\mu^{+} \mu^{-}$collisions at c.o.m. energies up to $4 \mathrm{TeV}$. We point out that the bileptonic gauge bosons predicted in some extensions of the Standard Model would be readily discernable from the backward scattering cross-section, for bilepton masses up to a substantial fraction of the c.o.m. energy.
\end{abstract}

Typeset using REVTEX 
In some extensions of the Standard Model, there occur additional gauge bosons with lepton number $L= \pm 2$ which are called bileptons. Some reviews of their properties are given in [1,2]. The detailed couplings of bileptons to fermions depend on the model. Generically they appear in $S U(2)$ doublets $\left(Y^{++}, Y^{+}\right)$and the charge conjugates, and couple to two leptons with a gauge coupling $g$ of order the electron charge $e$ but with the precise value depending on the model. In some models, bileptons couple also to quarks but this will not be relevant here.

One means of discovering bileptons which has been discussed [3, 4] is to look for the direct channel resonance in an electron linear collider in the $e^{-} e^{-}$mode. For a muon collider the $\mu^{-} \mu^{-}$mode could obviously be used similarly with no special advantage.

The principal advantage of a muon collider over an electron collider is that because of the much lower synchrotron radiation a "circular" machine can be used and c.o.m. energies up to several $\mathrm{TeV}$ can be foreseen. With this possibility, our purpose here is to point out what is quite simple: that for $\sqrt{s}>>M_{Y}$, and a coupling $g \geq e$ the backward scattering produced by bileptons can compete with the forward Coulomb peak. We shall illustrate this with the explicit lowest-order Born calculations of the cross-section and corresponding Figures for c.o.m. energies in the range $500 \mathrm{GeV}$ to $4 \mathrm{TeV}$ and bilepton masses $M_{Y}$ from $300 \mathrm{GeV}$ to $2 \mathrm{TeV}$.

The current lower limits on the bilepton masses are $M\left(Y^{+}\right)>230 \mathrm{GeV}$ from polarized muon decay [5] and $M\left(Y^{++}\right)>360 \mathrm{GeV}$ from muonium-antimuonium conversion [6].

The Feynman rule for coupling a doubly-charged bilepton to two like-sign muons arises from the interaction:

$$
\frac{g}{\sqrt{2}} Y_{\mu}^{++} \mu^{T} C \gamma_{\mu}\left(\frac{1-\gamma_{5}}{2}\right) \mu+\frac{g}{\sqrt{2}} Y_{\mu}^{--} \bar{\mu} \gamma_{\mu}\left(\frac{1-\gamma_{5}}{2}\right) C \mu^{T}
$$

In the $S U(15)$ model the value of the coupling is $g=1.19 e[7]$ while in the $331-$ model the coupling is somewhat larger: $g=2.085 e(=e / \sin \theta)$ [8]. We shall consider both possibilities.

The differential cross-section for $\mu^{+} \mu^{-} \rightarrow \mu^{+} \mu^{-}$can be written [7] 


$$
\begin{aligned}
\frac{d \sigma\left(\mu^{+} \mu^{-} \rightarrow \mu^{+} \mu^{-}\right)}{d \cos \theta}= & \frac{\pi \alpha^{2}}{2 s}\left[\left[\left|G_{L R}(t)\right|^{2}+\left|G_{R L}(t)\right|^{2}\right]+\left(\frac{t}{s}\right)^{2}\left[\left|G_{L R}(s)\right|^{2}+\left|G_{R L}(s)\right|^{2}\right]\right. \\
& \left.+\left(\frac{u}{s}\right)^{2}\left[\left|G_{L L}(s)+G_{L L}(t)\right|^{2}+\left|G_{R R}(s)+G_{R R}(t)\right|^{2}\right]\right]
\end{aligned}
$$

where

$$
G_{A B}(w)=\frac{s}{w}+\frac{g_{A} g_{B}}{e^{2}} \frac{s}{w-M_{Z}^{2}}+\left(\delta_{A B}-1\right)\left(\frac{g}{\sqrt{2} e}\right)^{2} \frac{s}{u-M_{X}^{2}}
$$

with $A, B=L, R$. For the Standard Model (SM) piece, $g_{L}=-\operatorname{ecot} 2 \theta_{W}, g_{R}=\operatorname{etan} \theta_{W}$. The SM cross section is obtained for $g=0$, the $S U(15)$ for $g=1.19 e$ and the 331 model for $g=2.085 e$.

The formulae for $e^{+} e^{-} \rightarrow e^{+} e^{-}$are identical to the above, so our focus on $\mu^{+} \mu^{-} \rightarrow \mu^{+} \mu^{-}$ at the highest c.o.m. energies is merely pragmatic.

In the above formulae, $s=\left(p_{1}+p_{2}\right)^{2}, t=\left(p_{1}-p_{3}\right)^{2}, u=\left(p_{1}-p_{4}\right)^{2}$ for $\mu^{+}\left(p_{1}\right)+\mu^{-}\left(p_{2}\right) \rightarrow$ $\mu^{+}\left(p_{3}\right)+\mu^{-}\left(p_{4}\right)$, and $\theta$ is the scattering angle between initial and final $\mu^{+}$in the c.o.m. frame.

The results are shown in the Figures. For the 331-model we have used $M_{Y}=300,500$ and $700 \mathrm{GeV}$ (in the simplest minimal version, $M_{Y} \leq 800 \mathrm{GeV}$ [9] although with an enlarged Higgs sector involving an octet of $S U(3)_{L}$ this bound can be relaxed). Figs. 1 and 2 then show the differential cross sections for $\sqrt{s}=1$ and $4 \mathrm{TeV}$, respectively.

For the $S U(15)$ model where $M_{Y}$ has no sharp upper bound, in Figs. 3 and 4 we have used $\sqrt{s}=1 \mathrm{TeV}$ and $\sqrt{s}=4 \mathrm{TeV}$, respectively. For $\sqrt{s}=1 \mathrm{TeV}$ we set $M_{Y}=300,500,700$ $\mathrm{GeV}$ and $1 \mathrm{TeV}$, while for $\sqrt{s}=4 \mathrm{TeV}$ we plot also $M_{Y}=1.5$ and $2 \mathrm{TeV}$.

All the Figures also show the Standard Model differential cross section.

For the relatively light bilepton masses $M_{Y}=300 \sim 700 \mathrm{GeV}$ we see from Figs. 1 and 2 firstly that the back-scattering effect is very large. Also, we see that, as expected, the distinction between the low masses may be easier at the low c.o.m. energy $1 \mathrm{TeV}$ than at 4 TeV. On the other hand, the ratio of the differential cross section of the 331-model to the Standard Model becomes larger as $\sqrt{s}$ is increased. 
Similar conclusions hold for the $S U(15)$ case: the overall effect is down by a factor $\left(g_{15} / g_{331}\right)^{4} \sim 0.106$ but is still readily distinguishable. Here bilepton masses up to 2 TeV (disallowed in the minimal 331-model) can be distinguished.

Finally, we mention polarized $\mu^{+} \mu^{-}$scattering. From Eq.(21) we see that $(d \sigma / d \cos \theta)$ for LR (= RL) polarizations is proportional to that for the unpolarized case in the backward direction for which $u=0$ and $t \simeq-s$, so there is no special advantage of polarization for this discovery process.

This work was supported in part by the U.S. Department of Energy under Grant No. DE-FG05-85ER-40219. 


\section{REFERENCES}

[1] F. Cuypers and M. Raidal, Nucl. Phys. B501, 3 (1997).

[2] F. Cuypers and S. Davidson, Eur. Phys. J. C2, 503 (1998).

[3] P.H. Frampton and B.H. Lee, Phys. Rev. Lett. 64, 619 (1990).

[4] C. A. Heusch, Int. J. Mod. Phys. A11, 1525 (1996).

[5] P.H. Frampton and E.D. Carlson, Phys. Lett. 183B, 123 (1992)

[6] R. Abela et al, Phys. Rev. Lett. 77, 1950 (1996)

[7] P.H. Frampton and D. Ng, Phys. Rev. D45, 4240 (1992).

[8] P.H. Frampton, Phys. Rev. Lett. 69, 2889 (1992);

F. Pisano and V. Pleitez, Phys. Rev. D46, 410 (1992).

[9] P.H. Frampton, hep-ph/9711281. 


\section{Figure Captions.}

1. Differential cross-section $d \sigma\left(\mu^{+} \mu^{-} \rightarrow \mu^{+} \mu^{-}\right) / d \cos \theta$ in 331-model for $\sqrt{s}=1 \mathrm{TeV}$ and bilepton masses $M_{Y}=300,500$, and $700 \mathrm{GeV}$.

2. Differential cross-section $d \sigma\left(\mu^{+} \mu^{-} \rightarrow \mu^{+} \mu^{-}\right) / d \cos \theta$ in 331-model for $\sqrt{s}=4 \mathrm{TeV}$ and bilepton masses $M_{Y}=300,500$, and $700 \mathrm{GeV}$.

3. Differential cross-section $d \sigma\left(\mu^{+} \mu^{-} \rightarrow \mu^{+} \mu^{-}\right) / d \cos \theta$ in $S U(15)$ model for $\sqrt{s}=1 \mathrm{TeV}$ and bilepton masses $M_{Y}=300,500,700 \mathrm{GeV}$ and $1 \mathrm{TeV}$.

4. Differential cross-section $d \sigma\left(\mu^{+} \mu^{-} \rightarrow \mu^{+} \mu^{-}\right) / d \cos \theta$ in $S U(15)$ model for $\sqrt{s}=4 \mathrm{TeV}$ and bilepton masses $M_{Y}=300,500,700 \mathrm{GeV}$ and 1, 1.5, $2 \mathrm{TeV}$. 


\section{FIGURES}

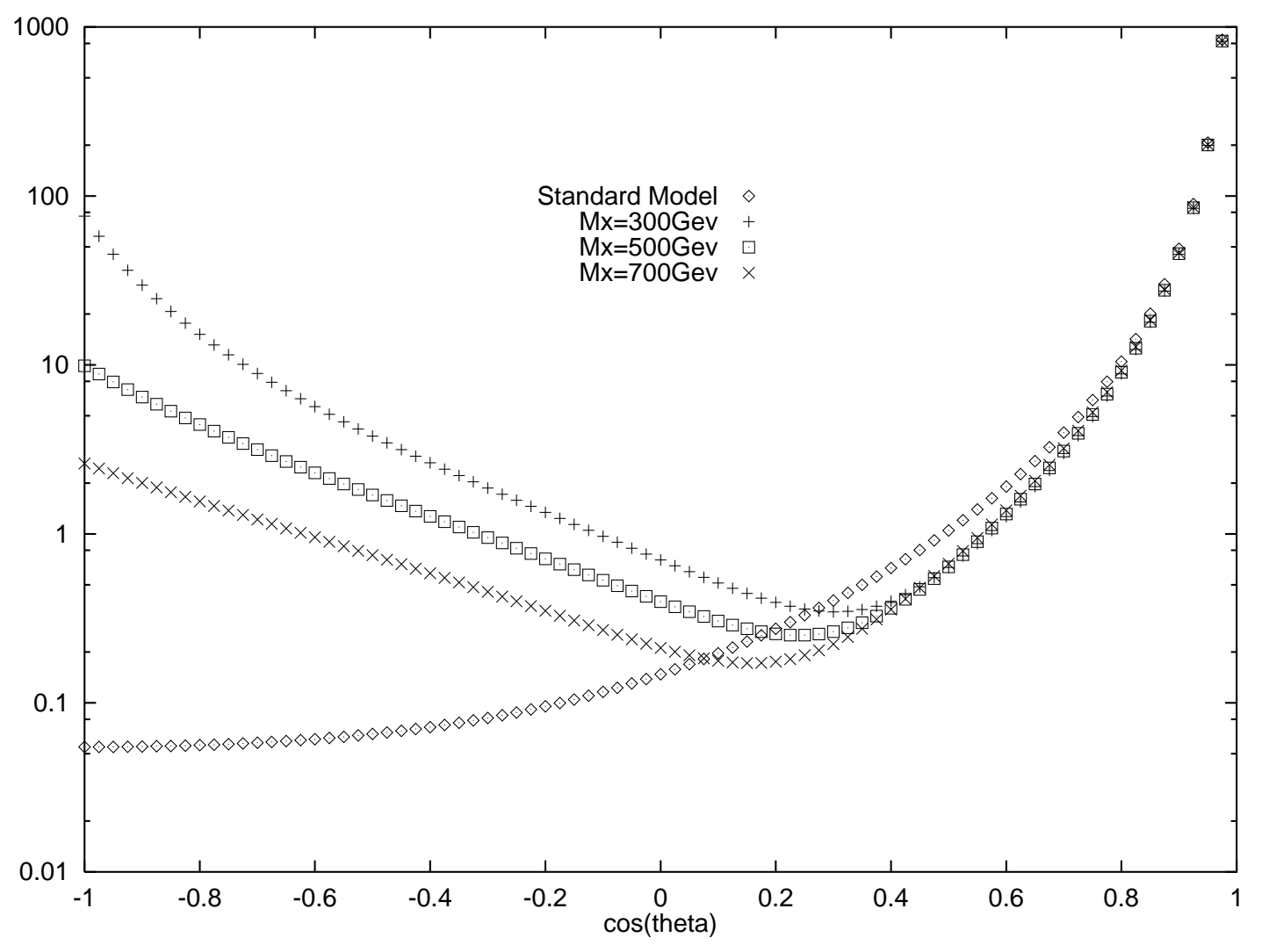

Fig. 1 


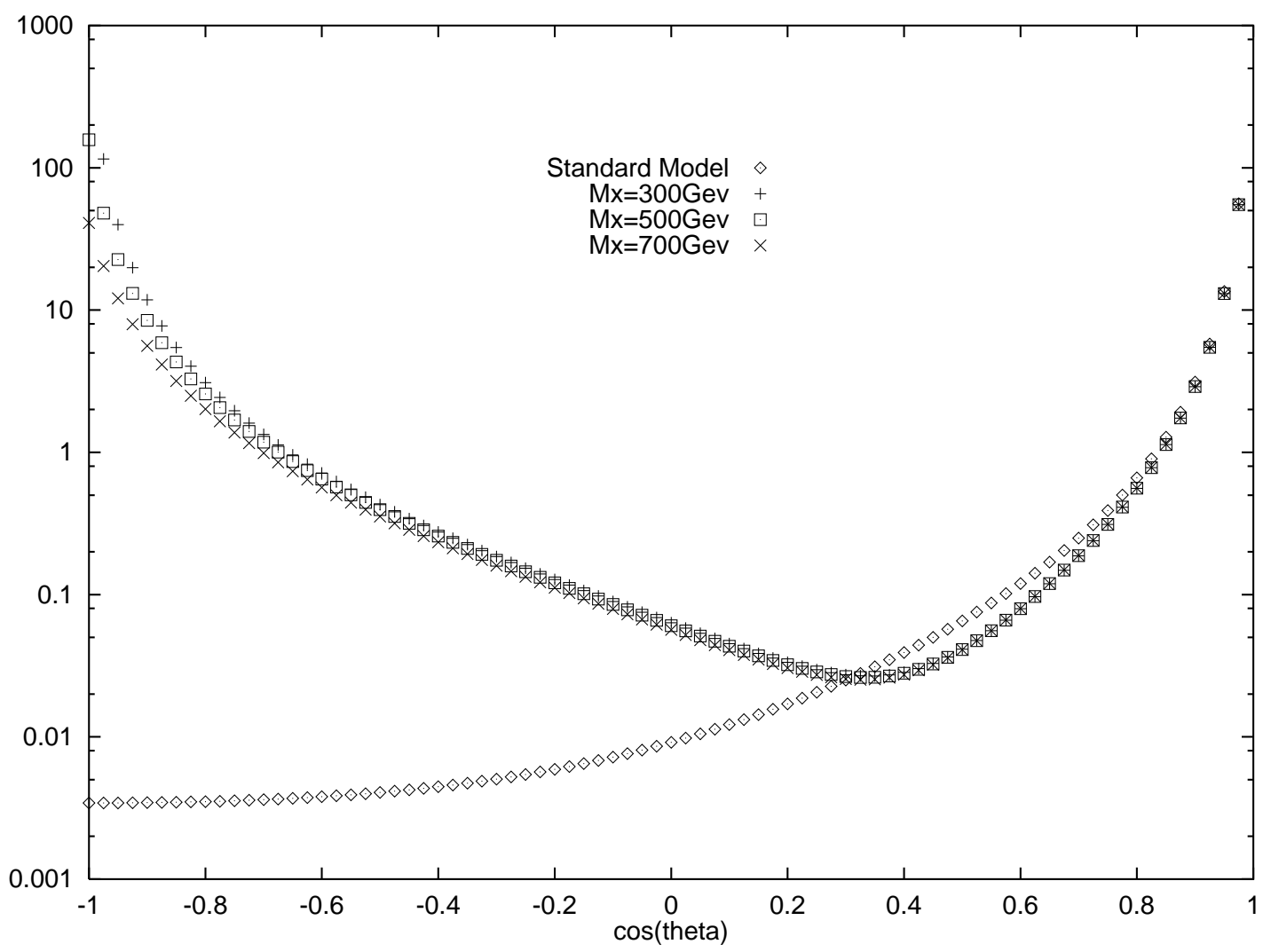

Fig. 2 


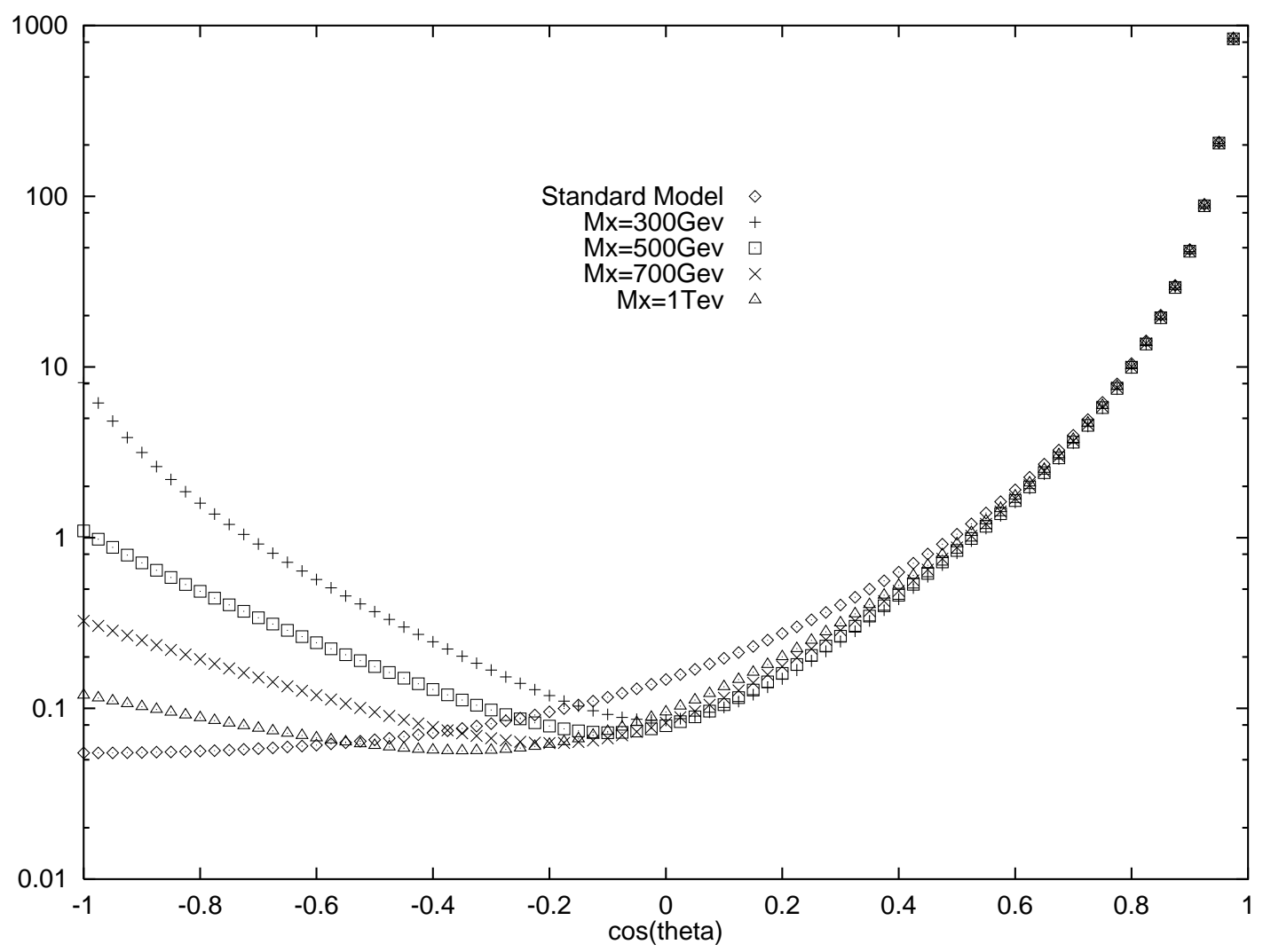

Fig. 3 


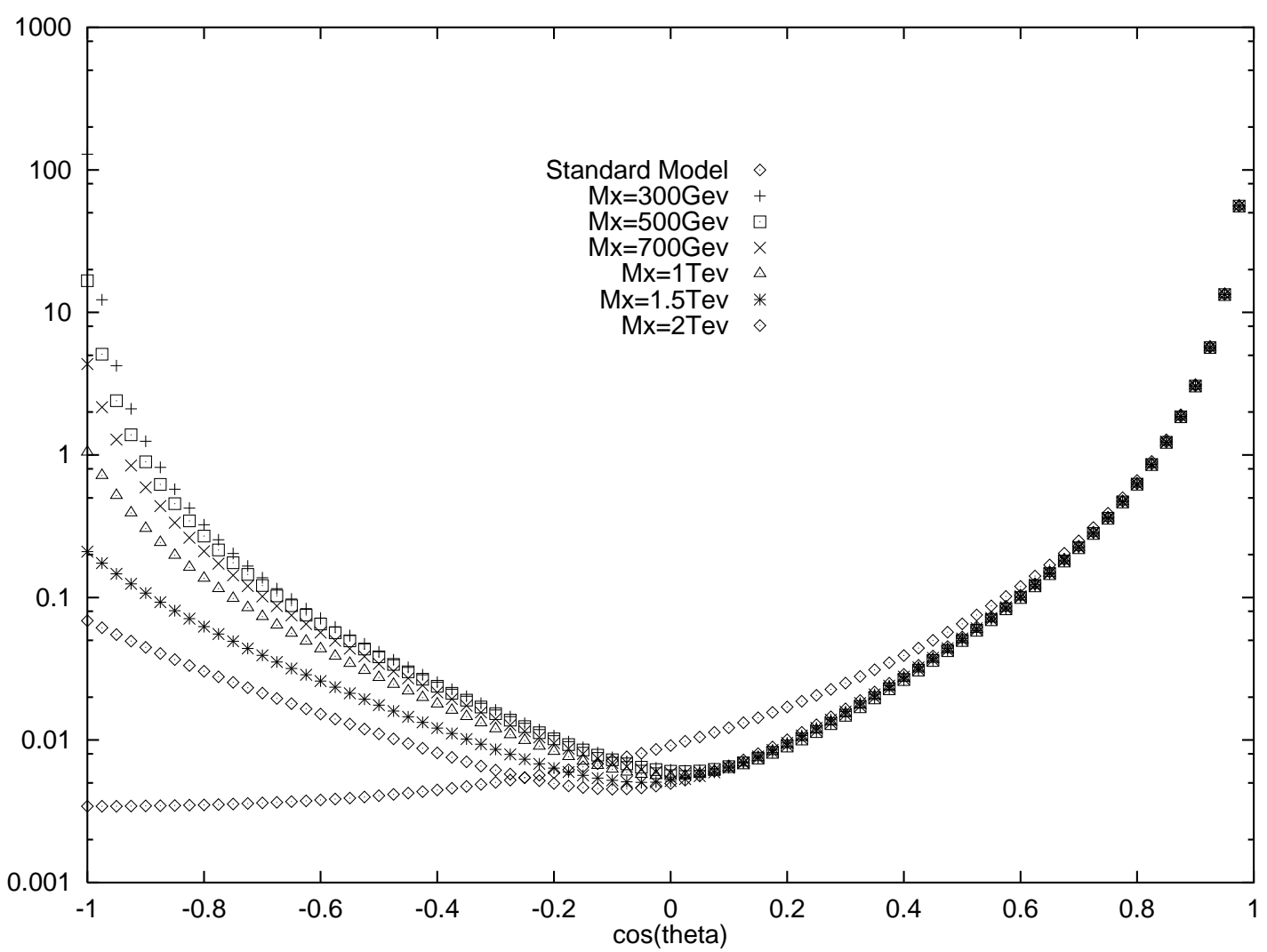

Fig. 4 